\title{
Effect of aspect ratio and anode location on the field emission properties of a single tip based emitter
}

\author{
R. C. Smith, ${ }^{\text {a) }}$ J. D. Carey, R. D. Forrest, and S. R. P. Silva \\ Nanoelectronics Centre, Advanced Technology Institute, School of Electronics and Physical Sciences, \\ University of Surrey, Guildford, Surrey, GU2 7XH, United Kingdom
}

(Received 24 September 2004; accepted 31 January 2005; published 6 April 2005)

\begin{abstract}
The effect on the field emission characteristics of the aspect ratio of an isolated emitter, together with the position of the anode electrode are reported. We show by computational simulation that the field enhancement factor $\beta$ is only dependant on the emitter height $h$, radius $r$, when the anode to cathode separation $D$ is greater than three times the height of the emitter away from the tip. In this regime the enhancement factor is independent of the anode location and approaches a value depicted by $h$ and $r$ alone and is described by the expression $\beta_{0}=(1+\sqrt{h / \alpha r})^{m}$ where $\alpha=2$ and $m=1$. As the anode is brought close to the tip of the emitter, the emitter tip and anode approximate a parallel plate configuration and the enhancement factor tends to unity. Extracted enhancement factor and threshold fields are described by a modified applied electric field taking $D-h$ as the separation. Comparison with previously reported experimental results is also given. (C) 2005 American Vacuum Society. [DOI: 10.1116/1.1880072]
\end{abstract}

Carbon nanotubes (CNTs), since their identification in 1991, ${ }^{1}$ have shown to possess a fascinating structure, and their use as electron sources in vacuum microelectronics and nanoelectronics has been widely reported. ${ }^{2}$ The mechanism of field-induced electron emission from a nanotube is understood to be due to the applied electric field undergoing an increase at the tip of the CNT, often referred to as the field enhancement factor $\beta$. For a single, isolated CNT, the value of enhancement factor is believed to be dependant on the length, radius, and type of structure, i.e., multiwalled (MWNT), singlewalled (SWNT), open or closed cap: This has been subject to several computational and experimental investigations. $^{3-8}$ Geometric enhancement is not just applicable to CNT but also exists in a number of other tip-based structures including: $\mathrm{SiC}$ nanowires, ${ }^{9} \mathrm{MoO}_{3}$ nanobelts, ${ }^{10}$ tungsten nanowires, ${ }^{11}$ spindt tips, ${ }^{12}$ and copper sulphide nanowire arrays. ${ }^{13}$ Much of the analysis performed on experimental data has relied upon analysis of the emission current $I$ to field $E$ (or voltage $V$ ) characteristics using the wellknown field emission mechanism of Fowler and Nordheim. ${ }^{14}$ The standard analysis often involves a plot of the $\log \left(I / E^{2}\right)$ versus $1 / E$ (or equally $\log I / V^{2}$ against $1 / V$ ) and from the slope of the graph an approximate value for the field enhancement factor $\beta$ can be extracted. The role of $\beta$ is the enhancement of the applied macroscopic electric field such that under the action of the local electric field, tunneling of electrons from the Fermi level, into the vacuum, through the potential barrier becomes possible. The interpretation of $\beta$, which is a dimensionless quantity if electric field rather than voltage is used in the analysis, is therefore of great importance.

There have been a number of attempts to model the behaviour of $\beta$ for a range of nanotube height and radius. Early work by Dyke and Dolan ${ }^{15}$ showed that for a planar anode

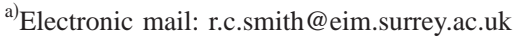

and a sphere-on-cone emitter the local field enhancement (neglecting $V / d$ ) close to the tip of the emitter was given by

$$
\beta_{L} \propto d^{1-n}
$$

where $\beta_{L}$ is the local field enhancement due to the emitter shape, $d$ is the emitter-anode gap, and $n$ is related to the cone opening angle (for an opening angle of 0 [sphere on a plane], $n=1$; for an opening angle of $\pi / 2$ [sphere on a cylinder], $n=0$ ). Mayer and colleagues ${ }^{16}$ have used a transfer matrix technique to compare the field emission behavior between metallic single-walled nanotubes (SWNT) and a multiwalled nanotube (MWNT) composed of the three single-walled tubes. They observed that the emission from the MWNT is larger than the total current obtained from consideration of the individual walls separately. They also noted that the emission was improved when the end of the tubes were convex rather than flattop. ${ }^{16}$ They further showed that the emission behavior of bundles of open and closed tubes were dependent on two factors; whether the tubes were open or closed and the role of proximity field screening by neighboring tubes. ${ }^{16}$ Closed tubes are found to emit less current than open tubes and that their field emission (FE) characteristics are found to be more sensitive to the local electric field. Theoretical investigations of field screening due to neighboring tubes are important in explaining the FE characteristics such as threshold field and emission site density. Nilsson et al. compare the threshold field for a mat of tubes and concluded that the optimum nanotube spacing was found to be approximately twice the height of the nanotube. ${ }^{17}$

The use of computer simulations continues to be an important and valuable tool in the electronics industry. Accurate calculations of electrostatic problems can be performed on a wide range of aspect ratios (height over radius) of emitters. In this article, electrostatic simulations of isolated emitters are reported. We investigate the electric field enhancement factor $\beta$ of a range of aspect ratios from 1 (being a hemi- 
sphere) to 500. We further explore the effects of the location of the anode electrode to emission characteristics.

Simulations of metallic emitters, to ensure no field penetration, of varying aspect ratio (height over radius) were carried out to investigate their field emission, and more particularly their field enhancement properties. The emitter consists of a hemispherical cap of radius $r$ on top of a cylindrical shank with total shank and cap height " $h$." They were placed in a vacuum and on a grounded cathode electrode so that the surface area of the emitter and the cathode has an equipotential value of $0 \mathrm{~V}$. An anode electrode was suspended above the emitter tip at a distance $D$ away. The commercially available SILVACO ${ }^{\mathrm{TM}}$ package $^{18,19}$ was employed to carry out the simulations, in two dimensions, and due to the simulation package, the work area or width of the simulation setup, was kept at $32 \mu \mathrm{m}$ to ensure no field screening due to edge effects. A mesh (or grid) is automatically determined by the software over the structure, where intersections between the $x$ and $y$ axes of the mesh determines a calculation point. The density of intersection points were then manually increased around the emitter tip to allow the local electric field $E_{\text {local }}$ to be accurately extracted. A mesh line spacing of $0.5 \mathrm{~nm}$ was used at and around the tip. To ensure the accuracy of the extracted electric field we simulated the electric field enhancement of a hemisphere and also mathematically calculated the enhancement for comparison. The field enhancement factor of a hemisphere in two dimensions was found by solving a Laplace equation, a value of 2 was calculated. A 2 $\mu \mathrm{m}$ radius hemisphere on a grounded plane in a vacuum with a planar anode was simulated and both applied and local electric field were extracted by the simulator. A field enhancement factor of 2.0 was found confirming the accuracy of our setup. Figure 1(a) is a schematic of the simulation setup. Current-voltage $(I-V)$ measurements were carried out on an emitter of height 2, 4 and $6 \mu \mathrm{m}$ and radius $200 \mathrm{~nm}$ with an applied electric field of $0-100 \mathrm{~V}_{\mu \mathrm{m}^{-1}}$ to ensure sufficient electron emission from a single tip. Simulations were carried out at an anode to tip separation $D-h$, of $0.1 \mu \mathrm{m}$ above the tip with emission current and local electric field recorded, until emission was no longer noticed. Figure 1(b) shows the threshold field $E_{T}$ for the three emitters of heights 2, 4, and 6 $\mu \mathrm{m}$ and constant radius $200 \mathrm{~nm}$. It is clear that $E_{T}$ is highest at low $D$, decreasing and saturating at high $D$. In this case, all properties of the emitter simulations were kept identical; the only aspect that was varied was $D$. To investigate further we extracted the electric field enhancement factor $\beta$ for a range of $D$.

Enhancement factor against $D$ is shown for an emitter of radius $100 \mathrm{~nm}$ and heights of 2, 4, and $6 \mu \mathrm{m}$ shown in Fig. 2(a) and radius $50 \mathrm{~nm}$ and heights 2, 4, and $6 \mu \mathrm{m}$ in Fig. 2(b). A range of $D$ from $0.1 \mu \mathrm{m}$ above the tip of the emitter, to $500 \mu \mathrm{m}$ are shown. It is appropriate to say at this point that while enhancement factor is usually defined as the local field over the applied field, where the applied field is usually taken as the applied voltage over anode to cathode separation $D$ or
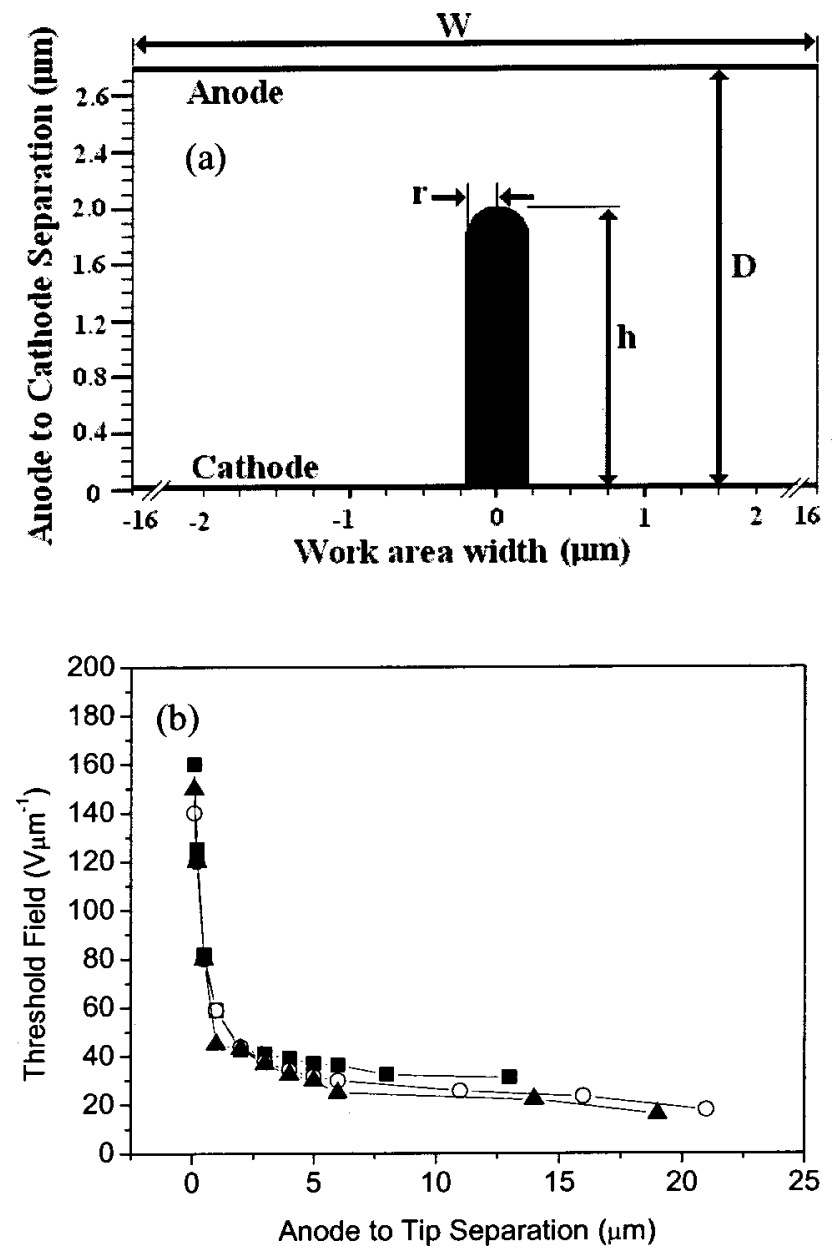

FIG. 1. (a) Schematic of the simulation setup showing a single isolated emitter in a vacuum on a grounded gold cathode electrode and beneath a gold anode with a positive potential. (b) Threshold field ET vs $D$ for emitters of radius $200 \mathrm{~nm}$ and heights of $2(\boldsymbol{\square}), 4(\bigcirc)$, and $6(\mathbf{\Delta}) \mu \mathrm{m}$.

$$
\beta=\frac{E_{\text {local }}}{\left(\frac{V}{D}\right)} .
$$

We feel that as the anode electrode approaches the tip of the emitter, the tip and anode approximates to a parallel plate configuration, as often assumed in probe-based field emission testing systems. In this case the applied electric field $E_{\text {applied }}$ can be taken as

$$
E_{\text {applied }}=\frac{V}{(D-h)} \text {. }
$$

Therefore, $\beta_{1}$ is defined as

$$
\beta_{1}=\frac{E_{\text {local }}}{\left[\frac{V}{D-h}\right]} .
$$

At sufficiently large anode to cathode separations the presence of the height of the emitter becomes negligible, however, when the anode is brought close to the tip of the emitter it can be said that the anode and tip approximate a parallel plate configuration. In this case it is more appropriate to define enhancement as $\beta_{1}$ in Eq. (4) due to when $D$ 

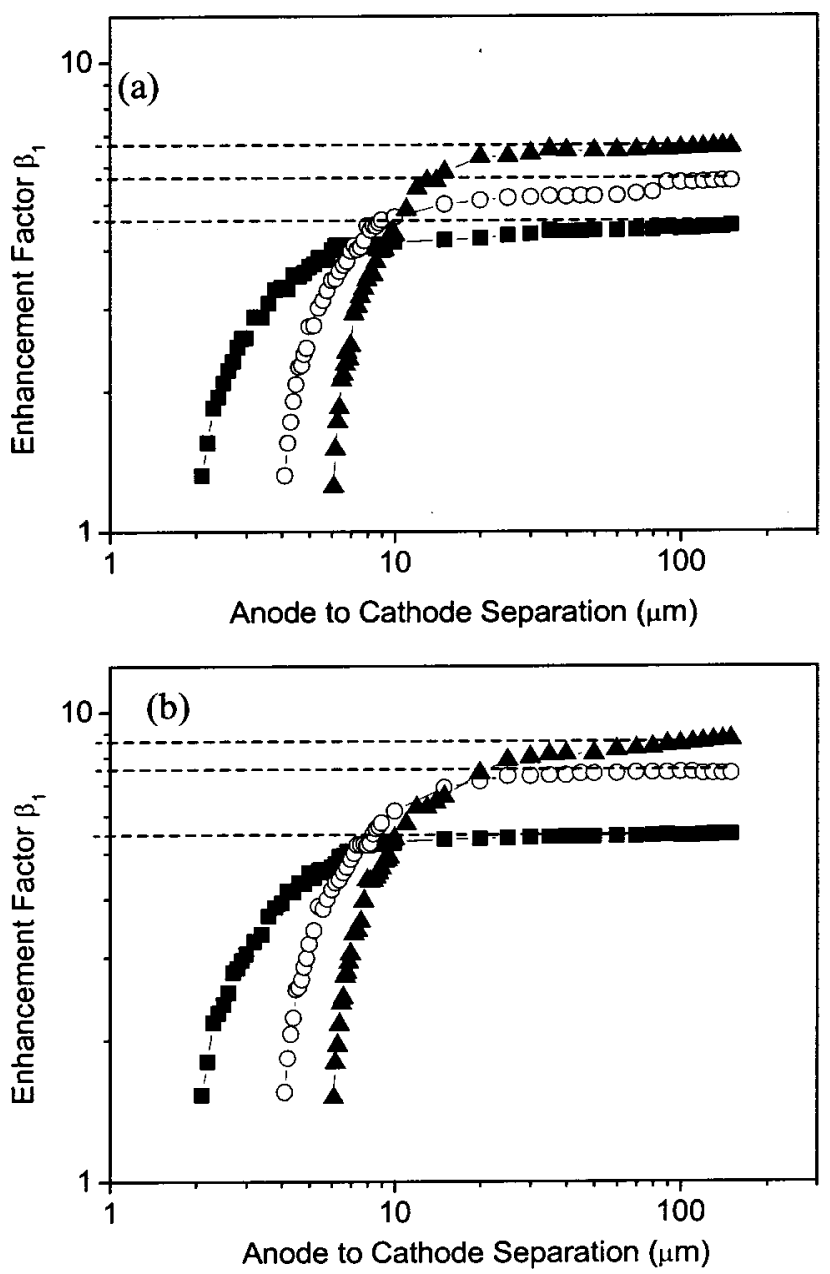

FIG. 2. (a) Enhancement factor $\beta_{1}$ trend with increasing $D$ for three emitters of constant radius $100 \mathrm{~nm}$ and heights of $2(\boldsymbol{\square}), 4(\bigcirc)$, and $6(\boldsymbol{\Delta}) \mu \mathrm{m}$. (b). Enhancement factor trend with increasing $D$ for three emitters of constant radius $50 \mathrm{~nm}$ and heights of $2(\boldsymbol{\square}), 4(\bigcirc)$, and $6(\boldsymbol{\Delta}) \mu \mathrm{m}$. Applied electric field is taken as $V /(D-h)$. Dotted lines indicate asymptotic fit to data using Eq. (6).

$-h$ approaches zero, $\beta_{1}$ approaches unity which is expected from a parallel plate configuration. It is seen in Figs. 2(a) and 2(b) that $\beta_{1}$ for all emitters is lowest at low $D$, increasing and saturating as $D$ increases. The point of saturation occurs at approximately $3 \mathrm{~h}$. For all emitters it is seen that $\beta_{1}$ approaches 1 as the anode approaches the tip. This ties in with the high $E_{\mathrm{T}}$ seen in Fig. 1(b) and also the assumption that the system approximates to a parallel plate configuration. Similar trends of threshold field and enhancement factor of tipbased emitters were seen in experimental results of singlewalled nanotubes by Bonard et al. ${ }^{20}$ They initially showed in a plot of current density against macroscopic field that as the anode electrode is close to the emitters, the threshold field is higher than that when the anode is sufficiently far away. Their results tie in with our simulated results of Fig. 1(b). They further went to extract a field enhancement factor for the sample and showed that it increases and saturates with an increasing interelectrode distance, which ties in with our simulated results of Figs. 2(a) and 2(b). It is also important to say from the graphs of Figs. 2(a) and 2(b) that at low $D, \beta_{1}$ is

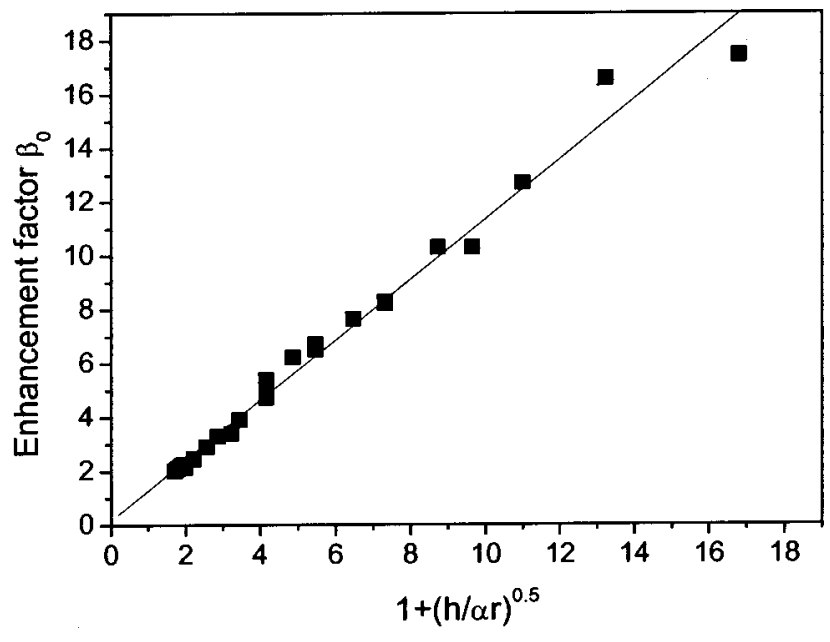

FIG. 3. $\beta_{0}$ against $1+(h / \alpha r)^{0.5}$. Linear fit has a gradient of 1 .

a function of $D, h$, and $r$, but at high $D, \beta_{1}$ is a function of $h$ and $r$ alone. These values at high $D$ are investigated in Fig. 3 . Aspect ratios of 1 to 500 were simulated, and $\beta_{0}$ extracted. Based on an analytical model for an ellipsoid cylinder, Kosmahl $^{21}$ derived an expression for the two-dimensional enhancement factor given by

$$
\beta_{0}=\left(1+\sqrt{h / r_{c}}\right)^{m}
$$

where $h$ is the height of the protrusion, $r_{c}$ is the radius of curvature given by $h^{2} / r$, and $m=1$. The fit to data from the simulation for a power law dependence based on Eq. (5) and $r_{c}=$ radius of the curvature taken gives a slope $m=0.94$. Although the geometry of the spheroid is different from that of a cylindrical shaft capped with a hemisphere, the excellent fit between the simulated data and Eq. (5) strongly indicates that the functional form of Eq. (5) is able to describe the data obtained in this study. An alternative equation with a similar functional form is given in Eq. (6) below

$$
\beta_{0}=(1+\sqrt{h / \alpha r})^{m} \text {. }
$$

In this case a value of $m=1$ is obtained with $\alpha=2.0$. It is not clear whether there is any significance that can be attached to a value of $\alpha=2$ at present. The values of $\beta_{0}$ for the range of aspect ratios of $1-500$ are plotted in the form of $\beta_{0}$ versus $\left[1+(h / \alpha r)^{0.5}\right]$ and shown in Fig. 3. This function is employed to ensure a gradient of 1 , therefore linking the equation to simulated values for values of enhancement factor when the anode electrode is sufficiently far away from the protrusion. Using the simulated emitters of Fig. 2(a) of height 2,4 , and $6 \mu \mathrm{m}$ with radius $100 \mathrm{~nm}$ a limiting enhancement factor of 4.16, 5.47, and 6.48, respectively, were found using Eq. (6), and for Fig. 2(b) emitters of height 2, 4, and 6 $\mu \mathrm{m}$ with radius $50 \mathrm{~nm}$ give a limiting enhancement factor of 5.47, 7.32, and 8.74, respectively. This asymptotic expression can be seen as the dotted lines to Figs. 2(a) and 2(b); these values for the individual emitters are the limiting values of enhancement factor when the anode plane is sufficiently far from the emitter tip, and is depicted by the emitter geometry alone, not the precise anode location. 
In conclusion, we have shown that while the aspect ratio of tip-based emitters play an important role in determining the geometric enhancement factor, the location of the anode electrode is equally as important. It is only when the anode is greater than three times the height of the emitter that its true potential as an emitter becomes possible. At low $D$, the emitters approximate to a parallel plate configuration and therefore lose their usually high enhancement factor. It has been shown that careful consideration as to the location of an electrode above the emitter has to be employed.

The authors would like to thank the EPSRC Portfolio Partnership and Carbon Based Electronics programs for funding this research.

${ }^{1}$ S. Iijima, Nature (London) 354, 56 (1991).

${ }^{2}$ A. G. Rinzler, J. H. Hafner, P. Nikolaev, L. Lou, S. G. Kim, D. Tománek, P. Nordlander, D. T. Colbert, and R. E. Smalley, Science 269, 1550 (1995).

${ }^{3}$ W. A. de Heer, A. Chatelain, and D. Ugarte, Science 270, 1179 (1995).

${ }^{4}$ Y. Saito, K. Hamaguchi, T. Nishino, K. Hata, and K. Tohji, Jpn. J. Appl. Phys., Part 2 36, L1340 (1997).

${ }^{5}$ J. M. Bonard, J. P. Salvetat, T. Stockli, L. Forra, and A. Chatelain, Appl. Phys. A: Mater. Sci. Process. 69, 245 (1999).

${ }^{6}$ C. J. Edgcombe and U. Valdre, J. Microsc. 203, 188 (2001).

${ }^{7}$ V. Filip, D. Nicolaescu, and F. Okuyama, J. Vac. Sci. Technol. B 19, 1016 (2001).
${ }^{8}$ C. H. Adessi and M. Devel, Phys. Rev. B 65, 075418 (2002).

${ }^{9}$ K. W. Wong, X. T. Zhou, F. C. K. Au, K. L. Lai, C. S. Lee, and S. T. Lee, Appl. Phys. Lett. 75, 2918 (1999).

${ }^{10}$ B. Li, Y. Bando, D. Golberg, and K. Kurashima, Appl. Phys. Lett. 81, 5048 (2002).

${ }^{11}$ Y-H Lee, C-H Choi, Y-T Jang, E-K Kim, and B-K Ju, Appl. Phys. Lett. 81, 745 (2002).

${ }^{12}$ C. A. Spindt, J. Appl. Phys. 39, 3504 (1968).

${ }^{13}$ J. Chen, S. Z. Deng, and N. S. Xu, Appl. Phys. Lett. 80, 3620 (2002).

${ }^{14}$ R. H. Fowler and L. W. Nordheim, Proc. R. Soc. London, Ser. A 119, 173 (1928)

${ }^{15}$ W. K. Dyke and W. W. Dolan, Adv. Electron. Electron Phys. 8, 89 (1956).

${ }^{16}$ A. Mayer, N. M. Miskovsky, and P. H. Cutler, Phys. Rev. B 65, 155420 (2002).

${ }^{17}$ L. Nilsson, O. Groening, C. Emmenegger, O. Kuettel, E. Schaller, L. Schlapbach, H. Kind, J-M. Bonard, and K. Kern, Appl. Phys. Lett. 76, 2071 (2000).

${ }^{18}$ SILVACO $^{\text {TM }}$, Silvaco Int., Santa Clara, CA.

${ }^{19}$ Modifications to the Fowler-Nordheim model had to be performed as this model was originally designed for use in MOSFET devices calculating emission current between silicon and silicon oxide layers. Therefore, the emission barrier is defaulted to $2 \mathrm{eV}$. For emission from CNT's into a vacuum an emission barrier of approximately $4.5 \mathrm{eV}$ is used. This is achieved by modifying the two variables, f.ae and f.be which control the Fowler-Nordheim model within the SILVACO package.

${ }^{20}$ J. M. Bonard, M. Croci, I. Arfaoui, O. Noury, D. Sarangi, and A. Châtelain, Diamond Relat. Mater. 11, 763 (2002).

${ }^{21}$ H. G. Kosmahl, IEEE Trans. Electron Devices 38, 1534 (1991). 